\title{
- Illusory rotation in the haptic perception of a moving bar
}

A M L Kappers ${ }^{1}$, Z M Kluit ${ }^{2}\left({ }^{1}\right.$ Utrecht University, Helmholtz Institute; ${ }^{2}$ Utrecht University;

e-mail: a.m.l.kappers@uu.nl)

Haptic matching of the orientation of bars separated by a horizontal distance leads to large systematic deviations (eg Kappers and Koenderink, 1999 Perception 28 781-795). A bar on the right side has to be rotated clockwise in order to be perceived as parallel to a bar at the left side. This finding leads to the following intriguing question which we investigated in this study: Will a bar moving from left to right in a fixed orientation be perceived as rotating counterclockwise? Blindfolded subjects had to touch a bar that moved from left to right or from right to left while it was rotating clockwise or counterclockwise with different speeds or did not rotate. For each trial they had to decide whether the rotation was clockwise or counterclockwise. From psychometric curves fitted to the data, we could determine that the results were consistent with the findings in the static case: A bar moving from left to right has to rotate clockwise in order to be perceived as non-rotating (and vice versa). In other words, a translating bar causes the illusory perception of a rotation.

[This research was supported by The Netherlands Organisation for Scientific Research (NWO)]

\section{- Orientation profiles of the trapezium and the square-diamond geometrical illusions}

J Ninio (Laboratoire de Physique Statistique, Ecole Normale Supérieure;

e-mail: jacques.ninio@lps.ens.fr)

In previous work, "orientation profiles" (describing how the strength of an illusion varies with its orientation in the plane) were determined for several variants of the Zöllner and the Poggendorff illusions [eg Ninio and O'Regan, 1999 Perception 28(8) 949-964]. The study is extended here to two other classical illusions. Illusion strengths were determined for ten subjects at 16 orientations on 4 variants of the trapezium illusion and 8 variants of the square-diamond illusion. The trapezium illusion was maximal when the bases of the trapeziums were horizontal, and minimal when they were vertical. The oblique sides, but not the bases, were essential to the illusion, suggesting the existence of a common component between the trapezium and the Zöllner illusion. The square-diamond illusion is usually presented with one apex of the diamond pointing towards the square. I found that when the figures were displayed more symmetrically, the illusion was reduced by one half. Furthermore, it is surpassed, for all subjects, by an illusion that goes in the opposite direction, in which the diagonal of a small diamond is underestimated with respect to the side of a larger square.

\section{- The material-size illusion-The influence of material properties on haptic perception of volume}

M Kahrimanovic, W M Bergmann Tiest, A M L Kappers (Utrecht University;

e-mail:m.kahrimanovic@uu.nl)

Numerous studies, in different modalities, have shown that perception of a particular object property can be influenced by other properties of that object, resulting in perceptual illusions. For example, subjects perceive, both visually and haptically, an extent filled with lines as longer than the same but unfilled extent, suggesting that surface texture has an effect on size perception. The present study investigated the influence of surface texture, thermal conductivity and temperature on haptic perception of the volume of objects that could fit in one hand. Blindfolded subjects were asked to explore pairs of cubes differing in their material properties and to select the one with the larger volume. The results showed that, counterintuitively, a smooth cube was perceived as being significantly larger than a rough cube of the same volume. Moreover, cubes with a higher thermal conductivity were perceived as larger than cubes with a lower thermal conductivity, and colder or warmer cubes were perceived as larger than cubes neutral in temperature. These results revealed that haptic volume perception is not veridical and that it is influenced by different material properties. The observed biases could be explained by cognitive processes involved in the integration of information from different peripheral receptors.

\section{- The ambiguous corner cube and related figures}

K Brecher (Boston University; e-mail: brecher@bu.edu)

There are many remarkable 2D ambiguous figures. Several striking instances of simple geometrical solids or more complex 3D ambiguous objects also exist. Most of these examples can be perceived in only two different ways: eg as up or down (2D Necker cube); as convex or concave (ambiguous tri-wall, intaglio, hollow mask); or as figure or (back)ground (Rubin's Vase). We have studied examples of $2 \mathrm{D}$ images and 3D objects that have three or more interpretations. A solid cube with one corner missing is one example that elicits (at least) three different interpretations. Most observers (viewing 